\title{
EL CONCEPTO DE «PERVERSION» SEXUAL EN LA MEDICINA POSITIVISTA
}

\author{
Rafael Huertas García-Alejo
}

«En la historia de la locura, las funciones sexuales entran frecuentemente en juego, los órganos de la generación se convierten en el punto de partida de ilusiones, alucinaciones, disturbios de la sensibilidad general, que hacen nacer preocupaciones singulares y que crean los más inauditos delirios. Las obras antiguas están repletas, en efecto, de súculos y de íncubos, de suciedades de diablos y de brujas, de las obscenidades del Sabat, pero poco a poco, el Demonópata se transforma y se convierte en el Perseguido. Todos los pertrechos de la brujería son sustituidos, por los progresos de la ciencia, los instrumentos del gabinete del médico y los aparatos, cada vez más numerosos, que ingeniosas aplicaciones introducen en nuestras propias moradas. El Demonópata y el Perseguido no son, clínicamente, más que un solo enfermo y marcan el segundo período del delirio crónico. Los desarreglos morbosos son, efectivamente, los mismos, la interpretación por el solo hecho de las nociones geneales difundidas entre las masas, cambia, y las influencias diabólicas son reemplazadas por los agentes químicos, las grandes fuerzas naturales, el magnetismo, la electricidad, el teléfono, etc., todo el arsenal de la industria moderna» (1).

Estas palabras, con las que Valentin Magnan comenzó su disertación sobre «Des anomalies, des aberrations et des perversions sexuelles» en la sesión de la Société Médico-psychologique del 26 de enero de 1885 , son, sin duda, un buen ejemplo del talante positivista de una medicina que, rindiendo culto a los avances de la ciencia y de la técnica, se sumó de manera decidida a la superación de viejas rémoras me- 
dievales (2) y a la "apropiación científica» de una cantidad considerable de comportamientos humanos que la nueva psiquiatría positiva se esforzó en etiquetar y clasificar.

En el caso de las llamadas «perversiones» sexuales, este proceso de «apropiación médica», según la acertada expresión de George LanteriLaura (3), esta medicalización de hábitos y comportamientos sexuales, respondió a un complejo proceso que culminó a finales del siglo XIX con las definitivas e influyentes obras de Krafft-Ebing (4) y Havellock Ellis (5), muy poco antes de que Freud, con sus Tres ensayos sobre la teoria de la sexualidad (1905), iniciara una forma diferente de entender e interpretar las distintas manifestaciones de la vida sexual. No pueden desdeñarse, sin embargo, aportaciones anteriores de gran interés que fueron constituyendo, poco a poco, todo un cuerpo de doctrina encaminado a incorporar al sexo entre los polos medicalizados de lo normal y lo patológico.

Dos fueron, en mi opinión, las vías fundamentales que siguieron los estudios médicos del siglo XIX en lo referente a las relaciones sexolocura. Dos caminos que, aunque se entrecruzan frecuentemente en su recorrido, conviene distinguir para los propósitos de esta comunicación. Por un lado, y como es suficientemente conocido, las primeras nosografías psiquiátricas contemplan la existencia de una «locura erótica" que se relaciona, en la mayoría de las ocasiones, con las alteraciones - aumento o disminución- del apetito sexual. Son las «neurosis afrodisiacas» de Pinel (6), la «monomanía erótica» de Esquirol (7), etc.

Pero, por otro lado, junto a la descripción más o menos prolija de estos cuadros, que se consideran alteraciones fisiológicas o psicológicas de unos hábitos sexuales considerados «normales», surgen aquellos actos o comportamientos en los que se persigue el placer sin que, en ningún caso, se pretenda ni exista la posibilidad de que la especie se reproduzca y que, rápidamente, se consideran fuera de la norma moral. El amor «perverso» - las "perversiones»- se sitúa así del lado de la esterilidad, del placer y de la patología, "conjugando la muerte, el gozo y la enfermedad, por oposición radical a la sexualidad normal donde debe buscarse la salud, el poco placer - toda vez que en muchos casos se trata de un deber conyugal-y la reproducción» (8).

Autores germanos y franceses fundamentalmente comenzaron a preocuparse, ya en la segunda mitad del siglo, por este tipo de "anomalías». La íntima relación que de inmediato se estableció entre la "perversión moral de los instintos» y la llamada «locura moral»y, sobre 
todo, con el concepto de degeneración favoreció, en gran medida, que un numeroso grupo de estudiosos de la patología mental -servidores fieles del orden burgués recién instaurado- dirigieran sus esfuerzos a aislar y describir cuadros nosológicos cuyas características principales fueran la alteración "perversa» del apetito o de la consecución del acto sexual, a la vez que la nueva medicina legal encontraba en la sexualidad «desviada» fértil terreno en la que proyectar sus inquietudes científicas y sus medidas de control social. No es de extrañar que sean, precisamente, un alienista -Valentin Magnan-y un médico legista -Ambroise Tardieu - los encargados de afianzar, en la Francia del segundo Imperio, todo un discurso «científico» que asume la doble vocación de la medicina burguesa de la época, una doble mirada con un ojo, clínico, puesto sobre la enfermedad y otro, justiciero, sobre el escándalo moral (9).

\section{La norma y la ley}

El Étude médico-légale sur les attentats aux moeurs (1857) de A. Tardieu constituye, sin duda, una de las obras pioneras en ese abordaje “científico»y "positivo» de los comportamientos sexuales y, en especial, de la llamada «inversión sexual». Hasta entonces las alusiones al tema en la literatura médica habían sido breves y escasas (10) y tampoco existía ninguna reflexión sistemática desde otras disciplinas - jurídica, demográfica, histórica, etc. - de suficiente envergadura. La obra de Tardieu llegaba, pues, a cubrir un vacío en un momento político y sociocultural preciso, porque ofreció soporte intelectual a un segundo Imperio dedicado a perseguir implacablemente a todo tipo de transgresores y poner fin, también en el plano sexual, a la relativa tolerancia de la monarquía de Julio. A este respecto, el comisario François Carlier, director de la "Oficina de costumbres» a partir de 1860, llegó a reconocer que «Entre 1850 y 1870 la represión fue tan severa que hubo momentos de verdadero pánico" (11).

Así, A. Tardieu acomete su estudio con todo el prejuicio del celoso guardián de las buenas formas burguesas. Se muestra comprensivo con los autores que le han precedido admitiendo que «la sombra que envuelve estos hechos, la vergüenza y el disgusto que inspiran, han alejado constantemente las miradas de los observadores» (12) y manifestando explícitamente que «He dudado, largo tiempo, en hacer entrar en este estudio el mundo repugnante de la pederastia, pero no podía evitar pen- 
sar que ella forma el complemento indispensable y al mismo tiempo la parte menos conocida. Estoy, pues, decidido no solamente a no:guardar silencio sobre este triste tema, sino también a tratarlo con la extensión que ningún autor ha ofrecido hasta ahora, ni en Francia ni en el extranjero" (13).

Labor pionera, sí, pero también peligrosos juicios de valor, constantes a lo largo de toda la obra y propiciadores de un inequívoco discurso que, alejándose de la clínica, acaba por centrarse en una ética sexual digna de la más genuina intolerancia victoriana: «No pretendo» - continua Tardieu - «hacer comprender lo que es incomprensible y penetrar en las causas de la pederastia. Está, sin embargo, permitido preguntarse si hay otra cosa en el vicio que una perversión moral (...). :El libertinaje sin freno, la sensualidad embotada pueden por sí mismas explicar los hábitos de pederastia en padres de familia y conciliar, con la complacencia de hombres o mujeres, estos arrebatos contranatura” (14).

Por otro lado, la utilización simbólica de la suciedad y de lo repugnante es un elemento constante tanto en la obra de Tardieu como en otras posteriores. Así, no sólo se insistirá en el papel "contaminante" de la suciedad fecal en el coito anal - que transciende, obviamente, de la simple «infección» biológica-, sino que, incluso en sus descripciones médicas, en sus propias historias clínicas, los autores mostrarán un cierto reparo, una cierta "mala conciencia", por no poder evitar -en aras de la ciencia - «que de mi pluma salga la infame ignominia de los pederastas» (15). Y así, al abordar el examen detenido y metódico de homosexuales y pederastas, la pluma del sabio, cargada con científica candidez, puede aventurarse en el fango del vicio y de las miserias humanas. Para conocer sus interioridades, el médico, jugando al etnólogo estudioso de "otras razas", no dudará en transcribir el lenguaje obsceno de los seres degradados, aun cuando, en un último intento de protección, los párrafos más escabrosos sean redactados en latín (16).

De este modo, la morbosa curiosidad de la «limpia» sociedad burguesa, ese eterno binomio de atracción-repulsión, se convierte, de la mano del médico, del experto, en persecución y tragedia porque, en definitiva, el conocimiento y el estudio de la sexualidad "perversa" se hace imprescindible para ayudar a las fuerzas del orden y de la justicia a yugular eficazmente la «infección», la suciedad y la ofensa. La norma moral se convierte rápidamente en ley jurídica... pero también en ley natural. 


\section{Sintomatología y estigmatología}

Como es sabido, la influencia de la Naturphilosophie sobre la obra de eminentes naturalistas románticos propició la aparición de importantes estudios embriológicos en busca de esa idea de unidad morfológica en biología que justificara la existencia de la "cadena del ser». En esta línea de pensamiento, las investigaciones de Étienne Geoffroy Saint-Hilaire sobre el desarrollo embrionario anormal representan el comienzo de lo que más tarde sería llamado «teratología experimental» (17). La manipulación de embriones y la subsiguiente creación de «monstruos», lejos de considerarse una práctica «antinatural», vino a confirmar la tan buscada «unidad de plan» en la naturaleza y contribuyó, en buena medida, al ansia de dominio de la naturaleza presente ya en la ciencia ilustrada. Y es que para dominar la naturaleza y someterla a los intereses del capitalismo en expansión, era importante acabar con las incertidumbres sobre el futuro de la raza humana haciendo que los «monstruos» no se considerasen elementos contrarios a la naturaleza sino partes integrantes de la misma.

Pues bien, en contraposición a esta teratología biológica que propone la reunificación de los seres vivos, se edifica una teratología humana investida por la sociedad burguesa de la función opuesta, esto es, de separar unos seres humanos de otros, porque la aparición de unos «monstruos» hechos hombres impone su aislamiento y clasificación. Surge así una nosología de la transgresión en la que se incluirán negros, locos, criminales, activistas políticos y, cómo no, «perversos». Los importantes cambios en el conocimiento de las ciencias biológicas que surgen a lo largo del siglo pasado hacen que la sociedad europea y norteamericana reaccione como temiendo las novedades que el evolucionismo científico presentaba. Racismo antropológico, somaticismo médico, persecución del anormal o del extraño, etc., son algunas de las principales aportaciones que el positivismo científico presenta. Los preludios de las crisis económicas y del neocolonialismo ponen en marcha mecanismos ideológicos que preparan el terreno.

De este modo, paralelamente a una sintomatología clínica surge una estigmatología física y psíquica que es preciso explorar y describir y que aparece como la consecuencia inmediata de la regresión del hombre a la animalidad. En lo referente a la sexualidad, esta idea es aplicada, por ejemplo, a los onanistas cuando se apunta que estos sujetos recuerdan «animales inmundos, monstruos ridículos, en fin, seres fantásticos que vienen a excitar la imaginación bajo posturas más o menos 
lascivas (...) produciendo sobre los órganos genitales la más viva irritación que determina la evacuación seminal acompanada de esas debilidades, de ese abatimiento general...» (18).

Con la pederastia este proceso de retrogradación llega a su punto más extremo. La descripción de Garnier es suficientemente elocuente: «... los pederastas, en el onanismo bucal, tragan el esperma aspirado creyendo así reemplazar el que ellos han perdido, están también los sodomitas que lamen el ano con la lengua, lo que hace eyacular sin ninguna acción manual. Un pederasta y sodomita, más activo que pasivo, delgado, histérico, me ha asegurado haber sido objeto de este hecho. El hombre-perro está, de este modo, consumado" (19).

Como vemos, este recordatorio constante del peligro que acecha a la especie humana de retornar a la animalidad pasa por la denuncia de «bestias humanas» (20) y seres monstruosos. Por un lado, se pretende identificar las posiciones adoptadas durante el acto sexual "perverso» (en cuclillas, de rodillas, "a cuatro patas», etc.) como propias de seres inferiores; lo mismo ocurre con muchas de las acciones realizadas (succionar, lamer, sorber, etc.); pero, por otro lado, quizá el más importante y efectivo argumento que se emplea es la falta de correlación entre órgano y función: la boca, la mano o el ano reemplazan a la vagina, la lengua sirve de pene, etc. Es decir, determinados órganos de la economía cumplen funciones que no les son propias, la relación estructura-función se rompe en unos seres que, transgrediendo las leyes naturales, se alejan del arquetipo humano.

En definitiva, existen multitud de descripciones que nos permiten deducir la existencia de esta estigmatología sexual a la que vengo refiriéndome, que se relaciona directamente con esos otros estigmas físicos lombrosianos con los que la antropología criminal pretenderá «diagnosticar» la delincuencia (21), y que entronca directamente con la gran doctrina psiquiátrica del positivismo, con la teoría de la degeneración.

\section{Perversos y degenerados}

Publicado el mismo año que la obra de Tardieu -en 1857- el Traité des dégénérescences de B.A. Morel inicia un nuevo modo de entender la medicina mental en la que el somaticismo y las influencias hereditarias acaparan el protagonismo etiológico de la locura (22). Un tímido pero inequívoco intento de psiquiatrización del perverso comienza a alumbrarse ya en este autor cuando incluye la «perversión del sentido 
genésico» en la segunda clase de alienados hereditarios - primera variedad de degenerados de la especie humana-, junto con diversas monomanías y otros delirios de actos y sentimientos sin alteración aparente de las facultades intelectuales (23). «Bajo el nombre de perversión de los instintos genésicos»-escribe- «se comprenden los hechos que pueden igualmente pertenecer a la locura y a la inmoralidad en sus límites más extremos" (24). Aunque en este caso, justo es reconocer que lo moral no se identifica con la ética impuesta por la clase dominante sino con el más firme respeto a la naturaleza y con la más decidida defensa del ideal utópico de una raza humana limpia, bella, sana y sabia.

No obstante, la medicalización definitiva de las perversiones sexuales debe buscarse en la obra de Valentin Magnan, entre otras cosas, porque fue capaz de aplicar un modelo reduccionista a la gran variedad de comportamientos sexuales y una unidad de interpretación basada directamente en la neurofisiología:

En 1882, J. M. Charcot y el propio Magnan publican una serie de artículos en los Archives de Neurologie que, con el título «Inversion du sens génital et autres perversions sexuelles» (25) continuan y matizan el intento moreliano e imponen un nuevo enfoque que difiere ostensiblemente del de Tardieu y los médicos legistas de la énoca porque establecen, sobre bases científicas perfectamente coherentes con el positivismo médico - el papel de la herencia, la presencia de patología psiquiátrica bien definida que motiva o complementa el acto perverso, etc.-, que los pacientes por ellos descritos, no son responsables de sus actos en el momento de llevarlos a cabo y entran de lleno en la jurisdicción del alienista. El artículo es de gran interés porque las técnicas de exploración ya no se limitan a la simple y detallada anamnesis del paciente sino a la investigación de sus antecedentes familiares y personales y a la búsqueda de estructuras patológicas que justifiquen sus actos. No menos interés tiene la utilización precoz del término perversión para designar al fetichismo, del que presentan dos casos, antes de que A. Binet lo describiera con todo detalle en 1888 (26) y antes, naturalmente, de que el psicoanálisis lo considerase como la más típica de las perversiones.

Pero es la comunicación de Magnan ante la Société Médico-psychologique aludida al comienzo de este breve ensayo, la que constituye el documento en el que mejor pueden estudiarse la ideas del psiquiatra de Sainte-Anne sobre estos temas. Fiel a la más pura ortodoxia positivista, considera que la clínica permanece en el nivel de las apa- 
riencias y que es la anatomofisiología la que otorga las claves necesarias para el dominio de la ciencia propiamente dicha. De este modo, para nuestro médico, la única forma de comprender los fenómenos perversos consistiría en considerarlos como alteraciones particulares del · Sistema Nervioso Central.

Influido muy claramente por la doctrina de las localizaciones cerebrales que Broca había establecido muy pocos años antes, Magnan sugiere un modelo anatómico y jerarquizado del comportamiento sexual. La sexualidad normal correspondería a un funcionamiento armonioso y equilibrado entre el arco reflejo espinal y los centros corticales. A partir de aquí elabora una clasificación de las perversiones sexuales en tres categorías siguiendo un modelo muy simple y una terminología anatómica:

a) Los perversos espinales serían aquellos sujetos en los que el arco medular funciona de manera autónoma sin la regulación de los centros superiores. El ejemplo más claro sería el onanismo del idiota, del oligofrénico profundo, que Magnan califica de «un acto instintivo puramente brutal» (27).

b) En los perversos espino-cerebrales posteriores el centro genitoespinal de Budge está controlado exclusivamente por la corteza cerebral posterior, retrorolándica, esto es, por el nivel de recepción cortical de estímulos tanto sensitivos como sensoriales. Esta perturbación se traduciría por anomalías muy variadas del comportamiento sexual: ninfomanía, satiriasis, pero también algunas formas de homosexualidad en las que la imagen de un sujeto del mismo sexo, identificado por un cortex posterior que funciona mal, determinaría un estado de excitación sexual. Algo parecido ocurriría con el exhibicionismo y, claro está, con otras alteraciones mentales no necesariamente relacionadas con la sexualidad y motivadas, en definitiva, por un funcionamiento disarmónico de los centros cerebrales que da lugar a alucinaciones o a delirios de diverso orden.

c) Finalmente, en los espino-cerebrales anteriores «el punto de partida del reflejo se produce en la corteza cerebral anterior, es una influencia psíquica, como en el estado normal, que se produce sobre el centro genito-espinal; pero la idea, el sentimiento o la inclinación están aquí pervertidos» (28). Ideas, sentimientos e inclinaciones que acaban relacionándose, una vez más, con el viejo y siempre socorrido concepto de moral insanity.

El modelo neuroanatómico así propuesto persigue, evidentemente, varios objetivos. Por un lado, al considerar la perversión sexual como 
una enfermedad, y no como un simple atentado contra las. buenas costumbres, se reclama la pertenencia de dichas conductas perversas a la jurisdicción del alienista propiciando un nuevo motivo de enfrentamiento, tan habitual a lo largo de todo el siglo, entre médicos y juristas (29). Por otro lado, la aportación magniana supone la superación de la semiología de los grandes clínicos y la búsqueda de las estructuras patológicas de los enfermos. Como bien ha indicado G. LanteriLaura, «si comparamos a Magnan con Laségue, por ejemplo, nos vemos obligados a reconocer que la manera como Lasègue procede al examen de los exhibicionistas está centrado en el acto mismo y queda, finalmente, bastante tautológico: su pertenencia a la medicina no es evidente y el magistrado puede no estar convencido de la existencia de la enfermedad. Con Magnan la tautología cesa: el interés no es exclusivo ni está limitado a los actos mismos, la clínica busca los elementos que establecen que estos actos entran en la categoría mórbida, garantizado no por ellos mismo sino por los signos de desequilibrio mental. La enfermedad deja de ser una simple manera de hablar, o una comparación analógica, es una alteración funcional del sistema nervioso central» (30).

En esta misma línea se sitúan los autores de los últimos años del siglo, quienes, matizaciones aparte, consiguen la total inclusión de la perversión sexual en el área de la preocupación del médico. Es el caso de B. Ball, autor de La folie érotique, en la que se deja constancia de que «la inversión sexual no es un vicio, ni una pasión inmoral, sino una alteración morbosa que tiene el carácter de impulsión, de instinto; es una tendencia instintiva y congénita, es la única manera por la que un individuo mal organizado puede manifestar su vida sexual» (31). Asimismo destaca, en la última década del siglo, el estudio de J. Chevalier Une maladie de la personalité. Inversion sexuelle (1893), donde, además, se comienzan a considerar causas ambientales y sociales, como la pederastia y el safismo profesional o por necesidad, que relaciona directamente la prostitución y el pauperismo, el miedo a contraer enfermedades venéreas, etc. (32):

Sin embargo, la clave neurológica, deliberadamente ignorante de causas ambientales o biográficas, será la que domine la producción científica de las postrimerías del siglo. Ello tiene, sin duda, sus ventajas y sus inconvenientes: la ventaja fundamental es que, como hemos visto, la sexualidad se libera, al menos en parte, del discurso moral. El principal inconveniente es, quizá, que esa liberación, en el fondo; no es más que una gran falacia ya que al producirse la patologización de la sexualidad; los mecanismos destinados a controlarla ganan en efi- 
ciencia porque determinados sujetos dejan de depender de las decisiones más o menos arbitrarias y siempre subjetivas del poder para convertirse en enfermos, en individuos que, científicamente, objetivamente, transgreden la norma moral y la norma natural. La falacia biologista se consuma, así, una vez más.

\section{NOTAS}

(1) Magnan, V. (1885): «Des anomalies, des aberrations et des perversions sexuelles», Annales médico-psychologiques 1 (2 serie): 447-472, pp. 447-448.

(2) Véase Colle, C. (1987): «Ballet pour une sorcière; jeus sur les théories sexuelles» Frénésie. Histoire Psychiatrie Psychanalise 4: 11-37.

(3) Lanteri-LaURa, G. (1979): Lecture des perversions. Histoire de leur appropiation médicale. Paris.

(4) Von Kraff-Ebing, R. (1893): Psychopathia Sexualis.

(5) Havellock Ellis (1897): Studies in the Psychology of sex.

(6) PINEL, Ph. (1789): Nosographie philosophique, ou la Méthode de l'analyse appliquée à la médecine. Paris. T. III. Pinel distingue dentro de estas "neurosis afrodisíacas" (Orden $\mathrm{V}$ de su clasificación), las masculinas: anafrodisia, satiriasis y priapismo; y las femeninas: ninfomanía e histeria. Sobre el concepto de neurosis en la obra de Pinel, véase Lopez Piñero, J. M. y Morales Meseguer, J. M. (1970): Neurosis y psicoterapia. Un estudio histórico. Madrid. pp. 48 y ss.

(7) Esouirol, J. E. D. (1838): Des maladies mentales condidérées sous les rapports médical, hygienique et médico-légal. Pars. T. I. p. 247.

(8) LANTERI-LauRa, G. (1979): p. 39.

(9) Sobre el papel que la medicina jugó en el establecimiento de la famosa doble moral burguesa, véase ARON J. P. y KEMPF, R. (1984): La bourgeoisie, le sexe et l'honneur. Paris.

(10) Se trata, fundamentalmente, de la descripción de casos aislados de interés médicolegal. A modo de ejemplo, CASPER (1852): Viol et pédérastie. Tirada aparte de Revue trimestrielle de médecine légale.

(11) Carlier, F.: Les Deux Prostitutions, Paris, p. 445.

(12) TARDieu, A. (1857): Étude médico-légale sur les attentats aux moeurs. París, p. 114.

(13) Ibid. p. 118.

(14) Ibid. p. 126.

(15) Ibid. p. 119

(16) La costumbre se mantiene durante muchos años; véase, por ejemplo, LaUPTS (1896): Tares et poisons. Perversion et perversité sexuelles. Paris.

(17) Véase Bodemer, Ch. W. (1973): «La embriología durante el romanticismo». En Lain, P.: Historia Universal de la Medicina. T. V, pp. 193-202.

(18) Garnier, P. (1889): Anomalies sexuelles. Paris, p. 520.

(19) Ibid., p. 487.

(20) Como se sabe, la utilización de la expresión «bestia humana» en la sociedad finisecular para designar a locos y criminales fue utilizada profusamente. La literatura contribuyó a ello de manera notable -recuérdese la insistente presencia del término en las novelas de Vicente Blasco Ibán̄ez o la famosa novela de Emile Zola titulada, precisamente, 
La bête humaine-. Sobre esta última puede verse Huertas, R. y PESET, J. L. (1985): «Psiquiatría, crimen y literatura: el criminal nato en el naturalismo zoliano", Revista de la Asociación Española de Neuropsiquiatría, 5: 132-150.

(21) Véase PESET, J. L. y PESET, M. (1975): Lombroso y la escuela positivista italiana. Madrid.

(22) Véase Huertas, R. (1987): Locura y degeneración. Psiquiatría y sociedad en el positivismo francés. Madrid.

(23) MoRel, B. A. (1857): Traité des dégénérescences. Paris.

(24) Morel, B. A. (1860): Traité des maladies mentales. Paris, p. 413.

(25) El trabajo fue publicado por primera vez en los núms. 7 al 12 de Archives de Neurologie en 1882, y reeditado un año más tarde en el Progrés Médical. El texto consultado es la reciente edición de la colección Insania de Ed. Frénésie (1987) con presentación de Gérard Bonnet. Merece la pena destacar que esta colaboración entre Charcot y Magnan puede considerarse como una reconciliación entre los dos médicos, enfrentados desde 1877 con motivo del concurso de una nueva cátedra de clínica de enfermedades mentales que Charcot otorgó a B. Ball impidiendo que Magnan se hiciera cargo de ella.

(26) BINET, A. (1888): Études de psychologie expérimentale. Le fétichisme dans l'amour. Paris.

(27) MaGnan, V. (1885): p. 448

(28) Ibid., p. 455.

(29) Véase Peset, J. L. (1983): Ciencia y Marginación. Barcelona.

(30) Lanteri-LauRA, G. (1979): pp. 51-52. Recordemos que Ch. Lasègue fue el primero en describir el exhibicionismo (LASÉGUE, Ch. (1877): «Les exhibicionistes», L'Union Médicale, 5: 710-714) y que Magnan retomó el tema años más tarde (MAGNAN, V. (1880): Des exhibicionistes. Paris). De ahí que sea relativamente fácil establecer comparaciones entre ambos médicos con respecto a este tema concreto. Sobre la aportación general de Magnan al campo de las perversiones sexuales puede consultarse también LANTERI-LAURA, G. y L' DEL PISTOIA (1970): «Le perversioni sessuali: un modello della loro riduzione psichiatrica nel pensiero di V. Magnan", Sistema Nervioso, 22: 26-51.

(31) BALl, B. (s.d): La folie érotique. Paris, p. 156. Paris.

(32) Chevalier, J. (1892): Une maladie de la personnalité. Inversion sexuelle. Lyon y 